\title{
Detection and recognition of brain tumor based on DWT, PCA and ANN
}

\author{
Zahraa Faisal, Nidhal K. El Abbadi \\ Computer Science Department, University of Kufa, Education College, Iraq
}

\begin{abstract}
Every day over 100 Person will be diagnosed with a primary brain tumor and many more will be diagnosed with a cancer. Medical image processing is the most defy and invention field specially MRI imaging technique that scans and capture the internal structure of human brain. where specific type diagnoses of brain tumor can be a complicated affair, making confirmation of its diagnosis essential. In this paper, initially, brain images are filtered to remove unwanted particles, then a new method for automatic segmentation of lesion area is carried out based on mean and standard deviation. Combining both solidity property and morphological operation to detection only tumor from segmented image. Mathematical morphology such as close used to join narrow breaks regions in an object, fill the small holes and remove small objects. Employed wavelet transform to extract features from images, followed by applying principle component analysis (PCA) to reduce the dimensions of features. Extraction 13 feature of statistical and textural features to use them as input to the Artificial Neural Network (ANN) for classification. The algorithm is trained with 20 of brain MRI images and tested with 45 brain MRI images. Accuracy for this method was encourage and reach nearly $100 \%$ in identifying normal and abnormal tissues from brain MRI images. The proposed algorithm compared with other simila works and their results was better.
\end{abstract}

Copyright $@ 2020$ Institute of Advanced Engineering and Science. All rights reserved.

\section{Corresponding Author:}

Nidhal K. El Abbadi,

Education College,

University of Kufa, Iraq.

Email: nidhalelabbadi@uokufa.edu.iq

\section{INTRODUCTION}

A brain tumor is a very dangerous-type among all life threatening diseases which is increasing drastically among the humans. Based on data obtained from international scientific institutions such as American Cancer Society (ASCO) [1], rate of death caused by cancer is increasing rapidly in the whole world [2]. Brain tumors can have various forms and sizes may appear at different locations, where brain tumor has become one of the main causees for increasing mortality among children and adults. It's found that the total number of people that hardship and dying from brain tumors has been increased by 300 for each year during past few decades. The primary diagnosis is frequently stated as a brain lesion. It can be cancerous (abnormal) or noncancerous (normal) [3]. When normal or abnormal tumors grow, they can cause the pressure inside the skull to increase. This can cause brain spoilage, and it can be life-menacing. Brain tumors are categorized as primary (Benign) or secondary (malignant) [4]. Primary brain lumps happen in around 250,000 people a year globally, making up less than $2 \%$ of cancers. In children little than 15 , brain tumors are second only to acute lymphoblastic leukemia as the most common form [5]. So that computer aided diagnosis for automatic detection of brain tumors through MRI can provide highly accurate reconstruction of original images of the tumor and improve the diagnosis speed. The brain magnetic resonance image (MRI) classification is one of the most important fields in medical image processing. Magnetic resonance (MR) 
images are a very useful tool to detect the tumor growth in brain but precise brain image segmentation is a difficult and time consuming process [6]. The manual classification of brain MRI image is not always gives careful results and a time consuming. Therefore, there is an important for automatic classification using different techniques. In these techniques the classifier first trained with known data (images) that belonging to number of classes, then the classifier became capable of classifying invisible images accurately into a particular class [7].

\section{LITERATURE SURVEY}

(M. Sasikala and N. Kumaravel, 2008) introduce wavelet based texture features to distinguaish between benign and malignante brain tumor. Texture features performance evaluated by using neural network. The feature optimizer problem is addressed by using a genatic algorithm as a search method [8].

(Swapnali Sawakare, and Dimple Chaudhari, 2014) present a k-mean clustring method to segment the tumor for MR image. The first step in decision making is focus on features extraction by using GLCM and PCA, while the second step is the classification of benign and malignanit brain tumor based on probabilistic neural network [9].

(Said Charfi et al., 2014) suggested a machine learning procedure for computer-aided detection for automatic recognition of brain tumor through MRI images. The proposed method is based on the following computational techniques; the histogram dependent thresholding for image segmentation, the discrete wavelet transforms for features extraction, then using PCA for reducing the dimensionality of the extracted features, and the feed forward back-propagation neural network to classify inputs into normal or abnormal. The accuracy of the classification on both training and test images is $90 \%$ which was considerably good [10].

(Nidhal El Abbadi et al., 2016) Suggested new algorithm for detection of brain tumor based on singular value decomposition (SVD). The algorithm first trained with normal brain MRI images, then in the second step the algorithm become capable to classify the brain MRI images into normal and abnormal image (that have a tumor). The accuracy of this algorithm was up to 97\% [7].

(Nilesh Bhaskarrao et al., 2017) proposed BWT and SVM techniques image analysis for MRI-based brain tumor detection and classification. In this method, accuracy of $95 \%$ was achieved using skull stripping which eliminated all no brain tissues for the detection purpose [11].

\section{MATERIAL AND METHODS}

\subsection{Two Dimensional Discrete Wavelet Transform(2D DWT)}

Wavelet transform is an effective tool for feature extraction from MRI brain images; because it allows analysis of images at various levels of resolution due to its multi-resolution analytic property. The multi-resolution representation can provide a simple method for expounding the information about images. The 2D discrete wavelet transform can decompose an image into four different resolutions of subbands, corresponding to low frequencies in the horizontal direction and high frequencies in the vertical direction LH (vertical details), high frequencies in the horizontal direction and low frequencies in the vertical direction HL (horizontal details), low frequencies in both directions LL (approximation image) and high frequencies in both directions $\mathrm{HH}$ (diagonal details) [12, 13].

\subsection{Principal Component Analysis (PCA)}

Superfluous features increase the calculation times and memory storage which at times causes some complexities in the tumor classification, and so it is required to minimize the number of features. The (PCA) was used since it efficaciously reduces the dimensionality of the data and therefore reduces the computational cost of analyzing new data. PCA is a Wonderful tool to reduce the dimension of a data set consisting of a large number of interrelated variables while keep most of the variations. It is performing by converting the data set to a new set of ordered variables according to their importance or variances. This technique has 3 effects: it orthogonalizes the components of the input vectors so that uncorrelated with each other, it orders the resulting orthogonal components so that those with the largest variation come first, and eliminates those components giving the least to the variation in the data set [14].

\subsection{Proposed Method}

The main steps of proposed method to segment Brain Tumor and diagnosis shown in Figure 1. 


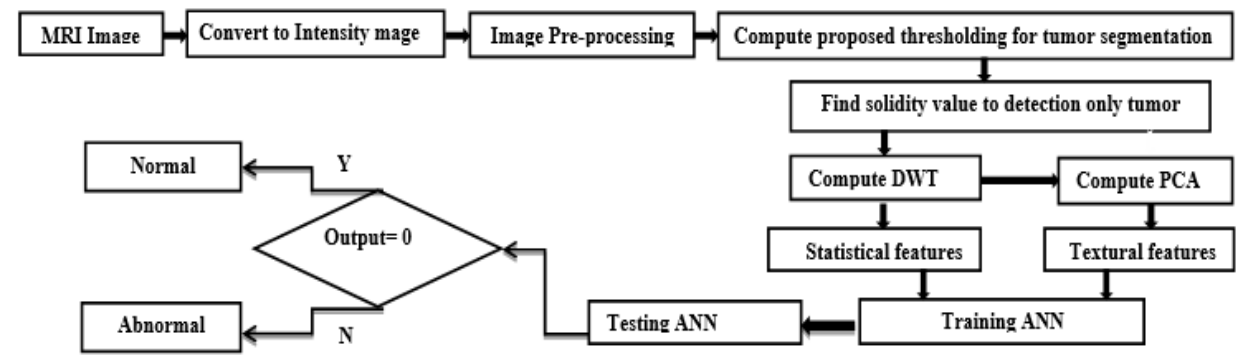

Figure 1. Flow chart for proposed method

Suggested method follows the following steps:

Step 1: Pre-processing

The presence of unwanted particles creates a poor segmentation; thus, incorrect identification of the tumor will negatively influence the results of classification step. In the current work, median filtering is suggested to improve the brain tumor MRI images and makes these images suited for future processing by clinical experts or imaging modalities. It also helps in improving parameters of MRI images. The parameters include enhancement the visual appearance of MRI images. Removing the irrelevant noise, and smoothing regions of inner part, maintaining relevant edges, and also enhance the contrast inside the image.

Step 2: Segmentation

Segmentation is a very important part and has a direct influence on the result of the classification. Indeed, poor segmentation will lead to a poor classification result even if the best classifier is used in the literature. A good segmentation will certainly lead us to a better classification rate even with a very basic classifier. However, perfect segmentation is difficult due to nature and technique beyond the MRI image. The proposed segmentation process based on the following steps:

1) The current method for tumor segmentation based on converts the color image to gray image. Find threshold (T) based on (Mean \& Standard Deviation) as in (1); to separate the image to two regions: one for tumor and the other for brain (background). The result image is binary image or can be color tumor with black background. This is can be achieved by using the following proposed (1).

$$
T=\left(\frac{1}{m n} \sum_{x=0}^{m-1} \sum_{y=0}^{n-1} f(x, y)\right)+\sqrt{\left(\frac{1}{m n} \sum_{x=0}^{m-1} \sum_{y=0}^{n-1}(f(x, y)-M)^{2}\right)}
$$

2) The solidity can be used to discriminate or detect tumor using shape criteria. Solidity is area fraction of the region as compared to its convex hull. The convex hull is what you'd get if you wrapped a rubber band around your region. It computed as:

$$
\mathrm{S}=\frac{\text { Area }}{\text { Convex Area }}
$$

The solidity for any convex object is 1 , while shapes like (T or E, etc.) would have a very low solidity [15]. From the experiment we found that the best segmentation can be achieved when threshold equal (0.4).

3) Removing the small objects and fill the small holes by using mathematical morphology such as close which used to join narrow breaks regions in an object. Figure 2 show the steps of segmentation.
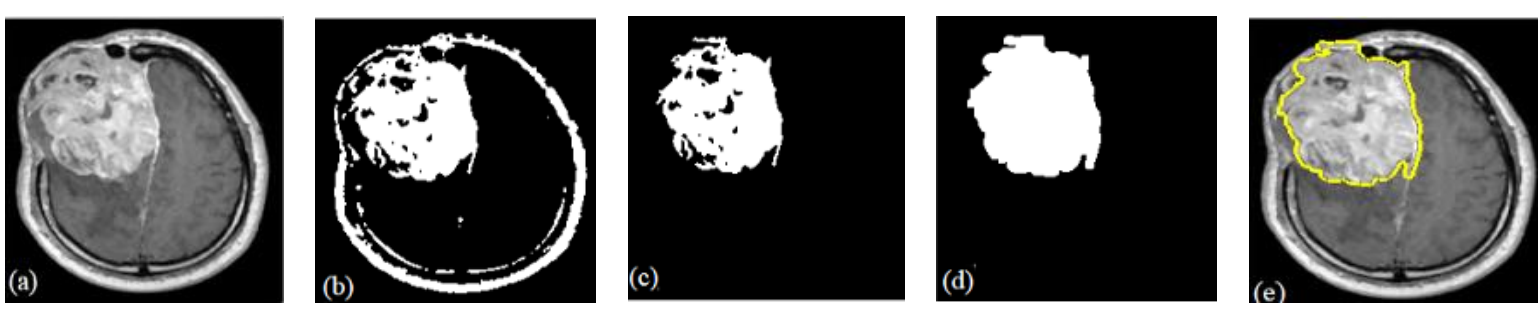

Figure 2. Phases of segmentation process of brain tumor, ((a) Image after pre-processing, (b) image after applying threshold, (c) image after apply solidity, (d) image after apply mathematical morphology, (d) edge detection by suggested method 


\section{Step 3: Features Extraction}

Feature extraction is the process of extracting the important details from segmented image such as texture, shape, contrast, and color features. The feature extraction process was carried out through two steps:

1) Feature Extraction Using (DWT \& PCA)

In this method, we used 3 level of (DWT) for extracting wavelet coefficients, followed by (PCA) to reduce the feature vector dimensions and increase the discriminative ability as shown in Figure 3.

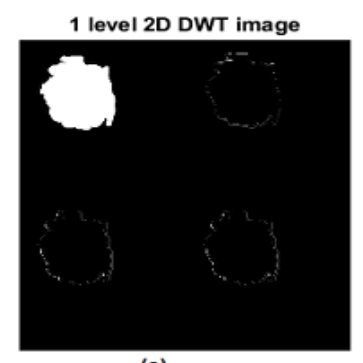

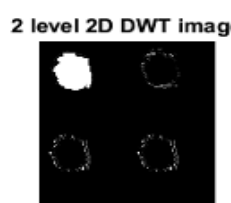

(b)

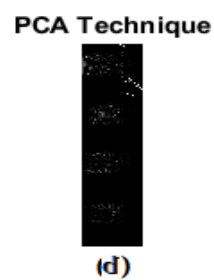

(a)

Figure 3. The phases of 3-level 2D DWT: (a) 1-level 2D DWT; (b) 2-level 2D DWT; (c) level-3 wavelet coefficients; (d) After apply PCA technique

The statistical features extracted are listed below:

a) Mean (M): The mean of image is calculated by summation all the pixel values in image divided by the total number of pixels in image [16].

b) Standard Deviation ( ): The standard deviation is the second central moment describing eventually distribution of an observed population and can use as a measure of inhomogeneity as in (3) [17].

$$
\sigma=\sqrt{\left(\frac{1}{m n} \sum_{x=0}^{m-1} \sum_{y=0}^{n-1}(f(x, y)-M)^{2}\right)}
$$

c) Mean of Variance (V): is a measurement of the spread between numbers in each image vector. The variance measures how far each number in the vector is from the vector mean. Variance is calculated by following (5) [16].

$$
\begin{aligned}
& \mathrm{V}_{\mathrm{i}}=\frac{1}{\mathrm{~m}-1} \sum_{\mathrm{i}=1}^{\mathrm{m}}\left(\left(\mathrm{A}_{\mathrm{i}}-\mathrm{M}\right)^{2}\right) \\
& \mathrm{V}=\frac{1}{\mathrm{n}} \sum_{\mathrm{i}=1}^{\mathrm{n}} \mathrm{V}_{\mathrm{i}}
\end{aligned}
$$

Where $\left(V_{i}\right)$ Variance for each vector in image, $\left(A_{i}\right)$ pixels in vector.

d) Entropy (E): is calculated to describe the randomness of the textural image as in (6) [17]

$$
E=-\sum_{x=0}^{m-1} \sum_{y=0}^{n-1} f(x, y) \log _{2} f(x, y)
$$

e) Skewness (Sk): is a measure of symmetry or the lack of symmetry. The skewness is computed as in (7) [17]:

$$
\mathrm{Sk}=\left(\left(\frac{1}{m n}\right) \frac{\sum(f(x, y)-M)^{3}}{\sigma^{3}}\right)
$$

f) Kurtosis $(\mathrm{Ku})$ : is a measure of the random variable's probability distribution. Is computed as in (8) [17]:

$$
\mathrm{Ku}=\left(\left(\frac{1}{m n}\right) \frac{\sum(f(x, y)-M)^{4}}{\sigma^{4}}\right)
$$

g) Inverse Difference Moment (IDM): is a measure of the local uniformity of an image. (IDM) may have a single or a range of values to distinguish between textured or non-textured [16]. 


$$
I D M=\sum_{x=0}^{m-1} \sum_{y=0}^{n-1} \frac{1}{1+(x-y)^{2}} f(x, y)
$$

h) Root-Mean-Square (RMS): is measurement that gives square root of the arithmetic mean of the squares of the values, or the square of the function that defines the continuous waveform [16].

$$
\mathrm{RMS}=\sqrt{\left(\frac{1}{m n} \sum_{x=0}^{m-1} \sum_{y=0}^{n-1} f(x, y)^{2}\right)}
$$

i) Smoothness (SM): is a property measured by the number of values it has that are continuous. It computed as in (11) [16].

$$
S M=1-\frac{1}{1+\left(\sum_{x=0}^{m-1} \sum_{y=0}^{n-1} f(x, y)\right)}
$$

2) Features extracted using GLCM

Texture analyses distinguish normal and abnormal tissues easily for machine learning and human visual perception. It also gives variation between malignant and normal tissues, which may not be visual to human eye. It improves the accuracy by choosing efficient quantitative features for early diagnosis. The textural features extracted are listed below [18] :

a) Contrast (Con): is a measure of intensity of a pixel and its neighbor on the image, as in (12).

$$
\text { Con }=-\sum_{x=0}^{m-1} \sum_{y=0}^{n-1}(x, y)^{2} f(x, y)
$$

b) Energy (En): defined as the quantifiable amount for repetitive pixel pairs. (En) is a parameter to measure the similarity of an image, as in (13).

$$
\mathrm{En}=-\sum_{\mathrm{x}=0}^{\mathrm{m}-1} \sum_{\mathrm{y}=0}^{\mathrm{n}-1} \mathrm{f}^{2}(\mathrm{x}, \mathrm{y})
$$

c) Homogeneity (Hom): is a measure the closeness of the elements distribution in the Gray Level Cooccurrence Matrix (GLCM) to the GLCM diagonal. (Hom) is 1 for a diagonal GLCM.

$$
\text { Hom }=\sum_{\mathrm{x}=0}^{\mathrm{m}-1} \sum_{\mathrm{y}=0}^{\mathrm{n}-1} \frac{\mathrm{f}(\mathrm{x}, \mathrm{y})}{1+|\mathrm{x}-\mathrm{y}|}
$$

d) Correlation (Cor): is measurement of spatial features dependencies between the pixels, as in (15).

$$
\operatorname{Cor}=\frac{\sum_{x=0}^{m-1} \sum_{y=0}^{n-1}(x, y) f(x, y)-M_{x} M_{y}}{\sigma_{x} \sigma_{y}}
$$

Step 4: Classification Using Artificial Neural Network (ANN)

ANN is capable to solve hugely complex problems due to the nonlinear processing capacities of its neurons [19]. The ANN is used for classifying brain tumor into normal or abnormal. The ANNs structure consists of three layers: input, output, and hidden. The output layer and hidden layer are adjusting weights value based on the error output in classification of various features. In this method, Back propagation (BPN) Algorithm are used for training as in Figure 4. After training, ANN can perform decision making. The inputs of ANN were 13 Feature while the outputs are one value as ( 0 or 1 ), 0 represents normal and 1 represents abnormal, as in Figure 5.

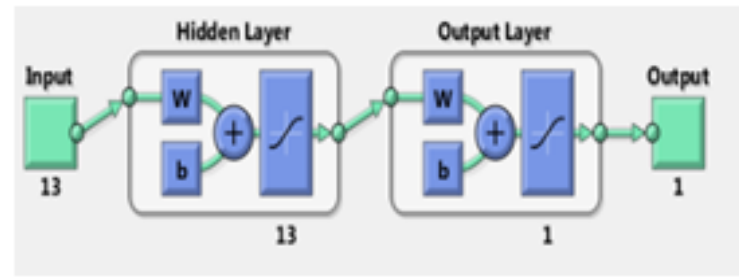

Figure 4. NN architecture

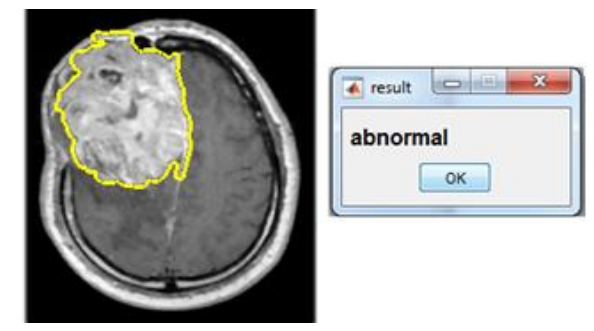

Figure 5. shows the result of the brain tumor 


\section{RESULTS AND DISCUSSION}

To test the performance of the proposed brain tumor diagnosis method we used 65 image, where 37 image are abnormal and 28 image are normal. The brain MRI images have been obtained from the websites (http://med.harvard.edu/AANLIB/, and DICOM dataset). The abnormal brain MRI images of the dataset consist of the diseases illustrated in Figure 6 (b-f) which represent (b-Alzheimer, c-glioma, d-sarcoma, e- meningioma, f-Pick's disease;). The proposed algorithm performance tested as follow:

a) Visually the proposed segmentation method works very well, where the tumor is determined only, while all the internal structures of the brain removed, as in Figure 7. The proposed method work very well in detecting the tumor, and ultimately in the segmentation process.

b) The performance of the proposed method is tested on 65 different images. Accuracy for this method was encouraging and reaches up to $100 \%$ in both of segmentation and diagnoses stage according to (16).

$$
\text { Accuracy }=\frac{\mathrm{TP}+\mathrm{TN}}{\mathrm{TP}+\mathrm{FP}+\mathrm{TN}+\mathrm{FN}}
$$

Where (TN) True negative, (TP) True positive, (FP) False positive, (FN) False negative.

c) We compared the segmented images resulted by proposed method with segmented images by other techniques. Figure 8 shows the comparing results for some technique, where (A. Original image. B. Fuzzy C-Mean technique. C. K-mean technique. D. Histogram technique. E. Watershed technique. F. Otsu technique. G. Proposed method). It is clear that the proposed method mpre effeicent for segmented the tumor, which will enhance the classification process.

d) After the segmentation process, we extract the features for each image (sample of images features listed in Table 1). According to the features values (the input to the ANN) we classified all images to normal or abnormal as (output of ANN). Figure 9 show sample of normal and abnormal image. These features have high impact on the classification process.

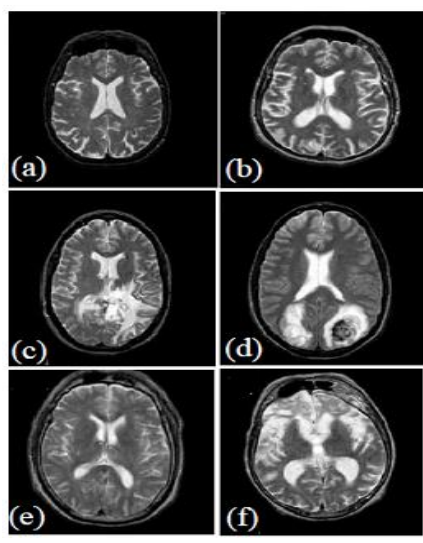

Figure 6. samples of the brain tumor: a) normal (b-f) abnormal

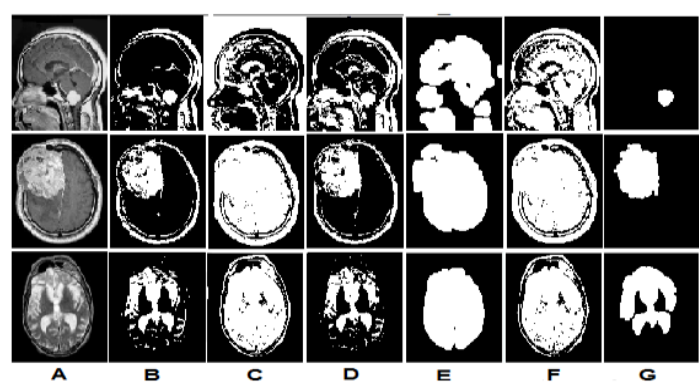

Figure 8. Comparing the tumor segmentation between proposed method and other techniques

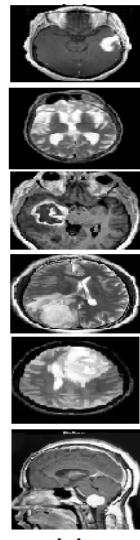

(a)

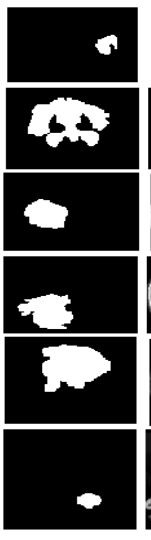

(b)

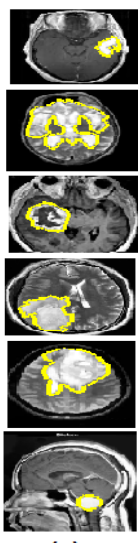

(c)
Figure 7. accuracy of suggested method. (a). Original image. (b). Segmented tumor (c). Edge detection

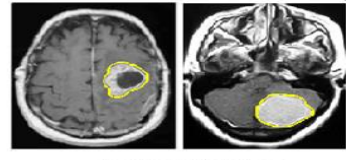

normal brains

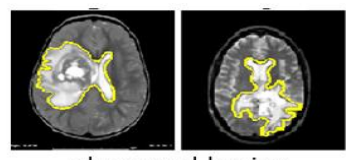

abnormal brains
Figure 9. sample of results of the proposed method 
Table 1. Features Values of Images in Figure (9)

\begin{tabular}{|c|c|c|c|c|c|c|c|c|c|c|c|c|c|c|}
\hline 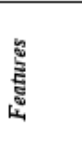 & $M$ & $\sigma$ & $V$ & $E$ & $S k$ & $\overline{K u}$ & IDM & $R m s$ & $S m$ & Con & $E n$ & Hom & Cor & 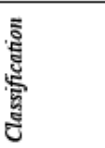 \\
\hline Imagel & 0.005 & 0.088 & 0.007 & 1.502 & 4.060 & 45.797 & 2.478 & 0.088 & 0.958 & 0.426 & 0.884 & 0.964 & 0.153 & Normal \\
\hline Image2 & 0.002 & 0.076 & 0.005 & 1.910 & 2.691 & 34.886 & 2.741 & 0.076 & 0.962 & 0.250 & 0.869 & 0.962 & 0.159 & Normal \\
\hline Image 3 & 0.005 & 0.085 & 0.007 & 2.022 & 3.169 & 36.616 & 1.693 & 0.085 & 0.950 & 0.358 & 0.871 & 0.961 & 0.141 & Abnormal \\
\hline Image4 & 0.004 & 0.094 & 0.008 & 2.047 & 1.756 & 18.930 & -.441 & 0.094 & 0.937 & 0.363 & 0.824 & 0.948 & 0.197 & Abnormal \\
\hline
\end{tabular}

e) The accuracy of proposed method compared with other methods, the results show in Table 2. It is clear that the proposed methed enhance the diagnosis of brain tumor and detect and classify all the tested images.

Table 2. Comparing Accuracy of the Proposed Method with the Similar Works

\begin{tabular}{|c|c|c|c|}
\hline Source & Year & Method_Name & Accuracy \\
\hline [13] & 2012 & Artificial neural networks design for classification of brain tumor. & $85.7 \%$ \\
\hline [5] & 2014 & A Novel Approach For Brain Tumor Detection Using Neural Network & $90 \%$ \\
\hline [11] & 2015 & $\begin{array}{l}\text { Brain MRI Image Classification Using Probabilistic Neural Network and } \\
\text { Tumor Detection Using Image Segmentation }\end{array}$ & $88 \%$ \\
\hline [14] & 2015 & $\begin{array}{l}\text { Brain Tumor MIR Image Segmentation and Classification Using by PCA } \\
\text { and RBF Kernel Based Support Vector Machine }\end{array}$ & $94 \%$ \\
\hline [4] & 2016 & Brain Tumor Classification Based on Singular Value Decomposition & $97 \%$ \\
\hline [15] & 2016 & $\begin{array}{l}\text { An Automatic Classification of Brain Tumors through MRI Using } \\
\text { Support Vector Machine }\end{array}$ & $98 \%$ \\
\hline \multirow[t]{2}{*}{ [6] } & 2017 & $\begin{array}{l}\text { Image Analysis for MRI Based Brain Tumor Detection and Feature } \\
\text { Extraction Using Biologically Inspired BWT and SVM }\end{array}$ & $96.51 \%$ \\
\hline & 2019 & Proposed method & $100 \%$ \\
\hline
\end{tabular}

\section{CONCLUSIONS}

In this paper, we used Artificial Neural Network to distinguish normal tumor from abnormal tumor. The contribution of proposed method was in convert image to binary image by using new suggested method to determine the threshold. Segmentation gives more accurate segmentation which help to increase the diagnosis accuracy of brain tumor images. Second contribution was in utilize each of discrete wavelet transform to extract features from images, and applying principle component analysis (PCA) to minimize the dimensions of features. The third contribution was in extracted each of statistical and textural features that distinguish between abnormal and normal tissues for help early diagnosis. The accuracy of detection brain tumor improves to $100 \%$ which is promised result comparing with other algorithms.

\section{REFERENCES}

[1] American Society of Clinical Oncology (ASCO), http://www.asco.org/

[2] Emre Dandıl1, Murat Çakıroğlu, and Ziya Ekşi, "Computer-Aided Diagnosis of Malign and Benign Brain Tumors on MR Images”, International Conference on ICT Innovations, ICT Innovations 2014, pp 157-166, Turky, 2014, DOI: $10.1007 / 978-3-319-09879-1 \_16$.

[3] Arakeri, Megha P., and G. Ram Mohana Reddy. "Computer-aided diagnosis system for tissue characterization of brain tumor on magnetic resonance images", Signal Image and Video Processing, vol. 9 no.2, pp. 409-425, 2015.

[4] N. V. Shree, Nandpuru, Hari Babu, S. S. Salankar, and V. R. Bora. "MRI brain cancer classification using support vector machine", Electrical Electronics and Computer Science (SCEECS), 2014 IEEE Students' Conference on IEEE, 2014.

[5] M. Mustaf, A. F. Sali, E. M. Illzam, A. M. Sharifa, and M. K. Nang, "Brain cancer : current concepts, diagnosis and prognosis”, IOSR Journal of Dental and Medical Sciences vol. 17, no. 3, pp. 41-46, 2018.

[6] M. Usman Akram, Anam Usman, "Computer aided system for brain tumor detection and segmentation", International Conference on Computer Networks and Information Technology", IEEE, Pakistan, 2011, DOI: 10.1109/ICCNIT.2011.6020885. 
[7] N. K. El Abbadi and N. E. Kadhim, "Brain Tumor Classification Based on Singular Value Decomposition", International Journal of Advanced Research in Computer and Communication Engineering vol. 5, no. 8, pp. 553-557, 2016.

[8] M. Sasikala, N Kumaravel, "Wavelet Based Automatic Segmentation Of Brain Tumors Using Optimal Texture Features”, 4th Kuala Lumpur International Conference on Biomedical Engineering 2008, Indonisia, pp 637-640, 2008, DOI: 10.1007/978-3-540-69139-6_159.

[9] Swapnali Sawakare1 and Dimple Chaudhari, "Classification of Brain Tumor Using Discrete Wavelet Transform, Principal Component Analysis and Probabilistic Neural Network”, International Journal for Research in Emerging Science and Technology, Volume-1, Issue-6, pp 13-19, 2014.

[10] S. Charfi, L. Redouan, and R. Lalitha. "A novel approach for brain tumor detection using neural network", International Journal of Research in Engineering and Technology, vol. 2, no. 7 pp. 93-104, 2014.

[11] N. B. Bahadure, A. K. Ray, and H. P. Thethi, "Image Analysis for MRI Based Brain Tumor Detection and Feature Extraction Using Biologically Inspired BWT and SVM”, international jornal of biomedical imagng, volum 2017, https://doi.org/10.1155/2017/9749108

[12] M. Gupta, G. Parmar, R. Gupta, "Discrete wavelet transform-based color image watermarking using uncorrelated color space and artificial bee colony", International Journal of Computational Intelligence Systems vol. 8, no. 2 pp. 364-380, 2015.

[13] M. Khalili, and A. David. "Colour spaces effects on improved discrete wavelet transform-based digital image watermarking using Arnold transform map", IET Signal Processing, vol.7, no.3, pp. 177-187, 2013.

[14] D. Ketkar. "Classification of Brain Tumors Using Principal Component Analysis And PNN Classifier", Proceedings of 12th IRF International Conference. Vol. 17. 2015.

[15] Haidekker, Mark, "Advanced biomedical image analysis", John Wiley \& Sons, 2011..

[16] P. N. D. Pergad and M. K. V Shingare, "Brain MRI Image Classification Using Probabilistic Neural Network and Tumor Detection Using Image Segmentation”, International Journal of Advanced Research in Computer Engineering \& Technology (IJARCET) vol. 4, no. 6, 2015.

[17] Nilesh Bhaskarrao Bahadure,Arun Kumar Ray, Har Pal Thethi, "Image Analysis for MRI Based Brain Tumor Detection and Feature Extraction Using Biologically Inspired BWT and SVM”, Int J Biomed Imaging. 2017; 2017 : 9749108, doi: 10.1155/2017/9749108.

[18] Kanchan Sharma, Priyanka, Aditi Kalsh, Kulbeer Saini, "GLCM and its Features", International Journal of Advanced Research in Electronics and Communication Engineering (IJARECE), Volume 4, Issue 8, pp 2180-2182, 2015.

[19] K. D. Kharat, P. P. Kulkarni, and M. B. Nagori, "Brain Tumor Classification Using Neural Network Based Methods", International Journal of Computer Science and Informatics vol.1, no.4, pp. 85-90, 2012.

[20] S. N. Deepa, and B. Aruna Devi. "Artificial neural networks design for classification of brain tumour." Computer Communication and Informatics (ICCCI) 2012 International Conference on. IEEE, 2012.

[21] P. Kumar and B. Vijayakumar, "Brain Tumour MRI Image Segmentation and Classification Using by PCA and RBF Kernel Based Support Vector Machine", Middle-East Journal of Scientific Research, vol. 23, no. 9, pp. 2106-2116, 2015. DOI:10.5829/idosi.mejsr.2015.23.09.22458

[22] M. Alfonse and A. M. Salem, "An Automatic Classification of Brain Tumors through MRI Using Support Vector Machine”, Egyptian Computer Science Journal, vol. 40, no. 3, pp. 11-21, 2016.

[23] .Devkota, Abeer Alsadoon, P.W.C. Prasad, A. K. Singh, A. Elchouemi, "Image Segmentation for Early Stage Brain Tumor Detection using Mathematical Morphological Reconstruction”, 6th International Conference on Smart Computing and Communications, ScienceDirect, Proceding Computer Science 125, pp 115-123, 2018, DOI: 10.1016/j.procs.2017.12.017.

[24] Parasuraman Kumar, B. VijayKumar, "Brain Tumor MRI Segmentation and Classification Using Ensemble Classifier", International Journal of Recent Technology and Engineering (IJRTE), Volume-8, Issue-1S4, June 2019.

[25] Shahariar Alam, et. al., 2019, “Automatic Human Brain Tumor Detection in MRI Image Using Template-Based K Means and Improved Fuzzy C Means Clustering Algorithm”, Big Data Cogn. Comput., 3, 27; doi:10.3390/bdcc3020027. 